\title{
IMPACTOS ECONÓMICOS INDUCIDOS POR LA DEMANDA FINAL EN SECTORES ESTRATÉGICOS PARA EL CAMBIO DE LA ESTRUCTURA PRODUCTIVA DEL ECUADOR
}

\author{
Carlos Ernesto Flores-Tapia \\ Pontificia Universidad Católica del Ecuador \\ Ecuador
}

Karla Lissette Flores-Cevallos Fundación Los Andes Ecuador 
Panorama Económico, Vol. 25 - No. 3 (Julio - Septiembre de 2017), pp. 4443-458

Carlos Ernesto Flores-Tapia

Karla Lissette Flores-Cevallos

\title{
Impactos económicos inducidos por la demanda final en sectores estratégicos para el cambio de la estructura productiva del Ecuador
}

\section{Resumen}

El cambio de la matriz productiva propuesta por el Gobierno Nacional de Ecuador, se basa en un modelo económico endógeno que tiene como rasgo distintivo básico su estructuración en torno a una función de producción donde la tasa de crecimiento depende básicamente del stock de tres factores productivos: capital físico, capital humano y conocimiento (o progreso técnico). Según Jaramillo (2014), existen sectores económicos estratégicos priorizados por el gobierno central para el cambio de la matriz productiva en los cuales se tienen oportunidades para satisfacer los requerimientos de los mercados interno y externo. El estudio contempla el análisis de impactos económicos en la producción y en el empleo, a partir de cambios en la demanda final de los productos seleccionados bajo tres alternativas de priorización de sectores estratégicos para el cambio de la matriz productiva nacional mediante el método insumo-producto, utilizando la herramienta de simulación desarrollada por el Banco Central del Ecuador (Banco Central del Ecuador, 2017a). La presente investigación contribuye al mejoramiento en la toma de decisiones relacionada con las políticas públicas productivas en Ecuador.

Palabras clave: Economía aplicada, Desarrollo Sostenible, Matriz Insumo Producto, Políticas Públicas, Herramientas de simulación, Productividad y Competitividad, Planificación.

Clasificación JEL: C67, E22, E23, E27, L52, O11, O21, O25, O41

\section{Economic impacts by final demand on strategic sectors for structural change in Ecuador}

\begin{abstract}
The change of productive matrix proposed by the National Government is based on an endogenous economic model whose basic feature is a production function where the growth rate depends on the stock of three factors of production: physical capital, human capital and knowledge (or technical progress). According to Jaramillo (2014), there are economic sectors prioritized by the central government to change the economic structure in order to satisfy the requirements of domestic and international markets. This research analyze the economic impacts on production and employment, by changes in the final demand of selected products in three alternatives of prioritization of strategic sectors for structural change through the input-output method, using the simulation tool developed by the Central Bank of Ecuador. The present research contributes to the improvement in the decision making related to productive public policies in Ecuador.

Keywords: Applied Economics, Sustainable Development, Input Ouput Analysis, Public Policies, Simulation Tools, Productivity and Competitiveness, Planning.

JEL Classification: C67, E22, E23, E27, L52, O11, O21, O25, O41
\end{abstract}

\section{Impactes économiques par la demande finale pour un changement structural en Équateur}

\footnotetext{
Résumé

Le changement de matrice productive du Gouvernement National de l'Équateur se base sur un modèle économique endogène. Cette matrice se base sur une fonction de production ayant comme taux de croissance axé sur trois facteurs productifs, capital physique, capital humain, et connaissance (progrès technique). Il existe des secteurs économiques stratégiques priorisés par le gouvernement central (Jaramillo 2014) pour changer le matrice productive afin de satisfaire les requis du marché interne et externe. Cette étude analyse les impacts économiques sur la production et le chômage, induits par le changement de demande finale selon des produits choisis. Trois alternatives de priorisation de secteurs stratégiques sont proposées selon la méthode consommation-produit. La Banque Centrale de l'Équateur a développé un outil de simulation (Banque Centrale de l'Équateur, 2017a). Cette étude aide à l'amélioration des décisions par rapport aux politiques publiques productives en Equateur.

Mots-clés: Économie appliquée, développement durable, matrice de saisie des produits, politiques publiques, outils de simulation, productivité et compétitivité, planification.

Nomenclature JEL: C67, E22, E23, E27, L52, O11, O21, O25, O41
} 


\section{Impactos económicos inducidos por la demanda final en sectores estratégicos para el cambio de la estructura productiva del Ecuador}

INFORMACIÓN DEL ARTÍCULO

Recepción de artículo: 07/02/2017

Concepto de evaluación: 30/04/2017

Aceptación de artículo: 06/05/2017
Carlos Ernesto Flores-Tapia* Pontificia Universidad Católica del Ecuador Ecuador

Karla Lissette Flores-Cevallos Fundación Los Andes Ecuador

\section{INTRODUCCIÓN}

El estudio contempla el análisis de los impactos económicos en la demanda final, la producción y el empleo, inducidos por variaciones en la demanda final de los sectores estratégicos priorizados por el Gobierno Nacional 2013-2017 (Secretaría Nacional de Planificación y Desarrollo, 2012), por el Banco Central del Ecuador ${ }^{1}$ y, en la Encuesta Nacional aplicada a la ciudadanía y sectores productivos del Ecuador ${ }^{2}$. En cada una de las alternativas se priorizan 30 productos de los contemplados en la nomenclatura internacional denominada Clasificación Central de Productos $(\mathrm{CPCN})^{3}$. Para la simulación de las variaciones se utiliza una herramienta informática desarrollada por el Banco Central del Ecuador, la misma que se sustenta en la Matriz de Leontief. (Nobel Prize, 2016).

El análisis de los impactos económicos inducidos por cambios en la demanda final de los sectores estratégicos para el cambio de la matriz productiva del Ecuador permitirá probar si la priorización de los sectores económicos que realiza el Gobierno Nacional es óptima ${ }^{4}$ o se pueden identificar otras propuestas de priorización con resultados mucho mejores,

\footnotetext{
* Autor para correspondencia

Correos electrónicos: cflores@pucesa.edu.ec*, fundacionlosandes@yahoo.es

1 Sectores denominados clave, motores y base, calculados con la metodología Rasmussen, publicados por el Banco Central del Ecuador en la Matriz Insumo Producto 2013.(Banco Central del Ecuador, 2014)

2 Encuesta a la ciudadanía aplicada a una muestra de 384 personas y encuesta aplicada a una muestra de 149 empresas. 3 CPCN (Banco Central del Ecuador, 2011)

4 De acuerdo a Hillier y Lieberman.(Hillier \& Lieberman, 2002) una característica de la investigación de operaciones es que intenta encontrar una mejor solución -llamada solución óptima-Se dice una mejor solución y no la mejor solución porque es posible que existan muchas soluciones que puedan considerarse como las mejores.
} 
como por ejemplo la identificación de los sectores clave de la economía ecuatoriana realizada por el Banco Central del Ecuador (Banco Central del Ecuador, 2014) utilizando el método de los coeficientes de Rasmussen (Sancho \& Cardenete, o 2014a), o el análisis de percepción de priorización de los sectores clave realizado por la ciudadanía y sectores productivos, resultante de la aplicación de una encuesta nacional.

El objetivo general del estudio es analizar los impactos económicos inducidos por cambios en la demanda final de los productos seleccionados para el cambio de la matriz productiva del Ecuador contemplados en tres alternativas de priorización. Los objetivos específicos son:

- Fundamentar teóricamente la investigación.

- Calcular los efectos en la demanda final, en la producción y en el empleo ocasionados por variaciones en los componentes de la demanda final, utilizando el método insumo producto.

- Analizar las implicaciones de las alternativas de priorización de los sectores estratégicos para el cambio de la matriz productiva del Ecuador.

A continuación se revisa la literatura previa y se redacta el estado del arte. Después se detalla la metodología. En la siguiente parte se exponen los resultados y la discusión de los mismos. Finalmente se presentan las conclusiones e implicaciones, así como las áreas de investigación futura.

\section{MARCO TEÓRICO}

\section{Matriz insumo producto}

"La matriz insumo producto (MIP) es una descripción de la economía de un país $\mathrm{y}$ un instrumento analítico generado a partir de la Tabla de Oferta y Utilización de Bienes y servicios (TOU). El objetivo de la MIP es ampliar el horizonte de la información producida por las Cuentas Nacionales, presentando en un solo cuadro las relaciones de la producción y los consumos intermedios de la economía". (Banco Central del Ecuador, 2014)

La matriz productiva (Secretaría Nacional de Planificación y Desarrollo, 2012) promocionada por el gobierno liderado por el Eco. Rafael Correa tiene como rasgo distintivo básico su estructuración en torno a una función de producción donde la tasa de crecimiento depende básicamente del stock de tres factores: capital físico, capital humano y conocimientos (o progreso técnico), que pueden ser objeto de acumulación y, además, generan externalidades. La matriz productiva gubernamental prioriza 14 sectores $^{5}$ y 5 industrias ${ }^{6}$ estratégicos para modelar el cambio (Secretaría Nacional de Planificación y Desarrollo, 2012).

Asimismo, el Banco Central del Ecuador tiene publicados los resultados de la Matriz Insumo Producto (Banco Central del Ecuador, 2014) correspondientes a los años 2007, 2010, 2012 y 2013 (Banco Central del Ecuador, 2017b); los cuales muestran las matrices de consumos intermedios; las matrices simétrica, de coeficientes técnicos, de demanda, la matriz inversa y los multiplicadores de producto y demanda; así como los encadenamientos directos y encadenamientos totales en los que se identifican los sectores clave,

5 1) Alimentos frescos y procesados 2) Biotecnología (bioquímica y biomedicina) 3) Confecciones y calzado 4) Energías renovables 5) Industria farmacéutica 6) Metalmecánica 7) Petroquímica 8) Productos forestales de madera 9) Servicios ambientales 10) Tecnología (software, hardware y servicios informáticos) 11) Vehículos, automotores, carrocerías y partes 12) Construcción 13) Transporte y logística 14) Turismo.

6 1) Refinería 2) Astillero 3) Petroquímica 4) Metalurgia (cobre) 5) Siderúrgica. 
motor, base e isla, utilizando el método de Rasmussen.

La investigación incorpora una tercera alternativa de priorización de sectores estratégicos para el cambio de la matriz productiva nacional resultante de la aplicación de una encuesta de percepción, tanto a la ciudadanía como a los sectores productivos del país ${ }^{7}$.

\section{Modelos multisectoriales}

Para la identificación de los sectores clave de una determinada economía se disponen de algunos Modelos Multisectoriales basados en las tablas input-output, o en las matrices de contabilidad social, o en modelizaciones más complejas relacionadas con Modelos de Equilibrio General Aplicado (Sancho \& Cardenete, 2014a); a saber, el método clásico de Rasmussen, el método de extracción hipotética, el método de la Matriz de Multiplicador del Producto y los modelos de equilibrio general aplicado (Sancho \& Cardenete, 2014b). Para el primero se utilizan las tablas input-output, para el segundo se pueden utilizar tanto las tablas input-output (TIO) como la matriz de contabilidad social (SAM), en los demás casos es necesario contar con la Matriz de Contabilidad Social (SAM), matriz que en este estudio no se contempla llevar a cabo. Los Modelos Multisectoriales permiten que los tomadores de decisión, particularmente en lo relacionado con políticas económicas, cuenten con herramientas y resultados para determinar los impactos de esas políticas en el territorio; por ejemplo en el empleo, el

\footnotetext{
7 Encuesta aplicada a la ciudadanía a nivel nacional: tamaño de la muestra 384, con un nivel de confianza del $95 \%$ y $5 \%$ de error, varianza 0.25 y un valor tipificado $\mathrm{z}$ de 1.96, doble cola. La muestra aplicada a los sectores productivos a nivel nacional es de 149 empresas.
}

producto interno bruto, el valor agregado, el gasto público, y la identificación de sectores clave para impulsar el desarrollo regional.

En el presente estudio, la alternativa de priorización de los sectores clave realizada por el Gobierno Nacional aplica una metodología participativa contemplada en la Ley Orgánica de Participación Ciudadana y en criterios relacionados con la potencialidad de los sectores priorizados para aprovechar la política de sustitución de importaciones y del comercio exterior (Asamblea Nacional del Ecuador, 2011; Ministerio Coordinador de la Producción, 2010). La identificación de sectores clave, motor y base contemplada en la publicación de la MIP del Banco Central del Ecuador utiliza el método de los coeficientes de Rasmussen, que a criterio de Banguero (2006) es el método más conocido y aplicado cuando se dispone de las tablas input-output. La tercera alternativa de priorización resultante de la aplicación de la Encuesta Nacional a la ciudadanía y sectores productivos emplea el muestreo estadístico sustentado en técnicas de investigación científica (Hernández Sampieri, Fernández Collado, \& Baptista Lucio, 2007; Lind, 2012).

Elmétodo deanálisisinput-output, también conocido como Matriz Insumo Producto, (Tarancón Morán, 2011) desarrollado por el profesor Wassily Leontief es un instrumento que permite medir las interdependencias de los diversos sectores económicos. Se fundamenta en la premisa de que el sistema económico responde a una complejidad de industrias mutuamente interrelacionadas, de tal manera que, toda industria provee su producción y recibe insumos o materia prima, a su vez, para su producción. De acuerdo a Schuschny (2005), el cálculo de 
la Matriz Insumo Producto con el método de la Matriz de Leontief se basa en los supuestos de homogeneidad sectorial, invarianza de los precios relativos, hipótesis de la proporcionalidad, hipótesis de aditividad, relación de precios presente en el año en que se elabora la matriz.

La Matriz de Leontief se presenta como herramienta idónea para el análisis macroeconómico, además su utilidad radica en la implementación de las mejoras en el marco contable del sistema de cuentas nacionales. Conjuntamente permite profundizar en la descripción y evaluación de las relaciones interdependientes entre los diferentes sectores productivos de la economía, principalmente todas aquellas transacciones intermedias reales entre los mismos. Sus datos usualmente son fáciles de ensamblar en los modelos macroeconómicos destinados a analizar las relaciones entre demanda final y los niveles de producción por actividades económicas. Es el caso, por ejemplo del Banco Central de Chile, (Banco Central de Chile, 2001), Banco central de la República Argentina, (Instituto Nacional de Estadística y Censos Argentina. Ministerio de Economía. Secretaría de Política Económica, 2001), Banco Central de la República Bolivariana de Venezuela, (Banco Central de Venezuela, 2012), Estados Unidos de Norteamérica, (Aroche, Frías, \& Torres, 2012), Banco Central de la República de Colombia, (Departamento Nacional de Estadística de Colombia, 2014), entre otros. El alcance que tienen dichos estudios es eminentemente descriptivo. (Flores-Tapia \& Flores-Cevallos, 2016). Para la medición de los impactos en la demanda final, la producción y el empleo de las distintas alternativas de priorización contempladas en este estudio se utiliza la herramienta informática de simulación del BCE, la misma que opera según el método de análisis input - output sustentado en la matriz de Leontief.
En el caso ecuatoriano, se destacan los siguientes estudios que utilizan la matriz insumo producto: "Elaboración de la matriz insumo producto y cálculo de los coeficientes de Rasmussen para la provincia de Cañar para el año 2007" (Campoverde \& López, 2014), "Análisis de impacto en la economía ecuatoriana por shocks exógenos en el sector agrícola (mediante el método input - output para el año 2007)" (Banderas \& Hidalgo, 2013), "Análisis input-output: identificación de los encadenamientos productivos y los sectores clave de la economía ecuatoriana para el año 2007" (Fernández, 2007) y, "Herramientas para simulación de matrices productivas óptimas del Ecuador" (Flores-Tapia \& Flores-Cevallos, 2016).

Algunos de los estudios iberoamericanos antes indicados, así como los destacados en el caso ecuatoriano identifican los sectores económicos clave y el análisis intersectorial; sin embargo, a diferencia de esta investigación, no contrastan alternativas de priorización ni miden los impactos de las variaciones en los componentes de demanda final en la misma demanda final, la producción y el empleo, ante las alternativas posibles de priorización de sectores estratégicos para el cambio de la matriz productiva del Ecuador.

\section{Encadenamientos sectoriales}

En el escenario nacional, el Banco Central del Ecuador publica los resultados del cálculo de la Matriz Insumo Producto (Banco Central del Ecuador, 2014) correspondiente al año 2013, se muestran en las matrices simétrica e inversa los valores de los cálculos de las interrelaciones sectoriales utilizando la Matriz de Leontief. Los resultados describen sintéticamente la situación de la economía nacional y cuantifican los efectos directos e indirectos que las industrias 
tienen sobre la economía del país, con el fin de facilitar y ser una herramienta en la toma de decisiones de la política pública.

En la identificación de los sectores clave en la matriz Insumo producto del año 2013 que se utiliza para este estudio, el Banco Central del Ecuador aplica el método de los coeficientes de Rasmussen, los cuales permiten clasificar los sectores de una economía en: 1) sectores base, cuando los encadenamientos hacia atrás son $<1$ y los encadenamientos hacia adelante son $>1$; sectores clave, aquellos en los que sus encadenamientos hacia atrás y hacia adelante son mayores a los generados en promedio por la economía; 3) sectores isla, cuando los encadenamientos hacia adelante y hacia atrás son $<1 \mathrm{y}$; 4) sectores motor, aquellos en los que los encadenamientos hacia atrás son mayores y los encadenamientos hacia adelante son menores a los generados en promedio por la economía (Banguero, Duque, Garizado, \& Parra, 2006; Sancho \& Cardenete, 2014b).

Según Soza \& Ramos (2005), los coeficientes de Rasmussen cuantifican los efectos hacia atrás $\left(\mathrm{BL}^{\mathrm{R}}\right)$ y delante $\left(\mathrm{FL}^{\mathrm{R}}\right)$ que puede experimentar un sector mediante la matriz inversa de Leontieff, así como valorar el aporte que hace cada sector a la economía en estudio y la interrelación sectorial; se determina entonces el efecto que produce el cambio en una unidad monetaria en la demanda final de cada sector en el producto total de todas las ramas. En las aplicaciones de los coeficientes de Rasmussen se distingue el "poder de dispersión" como la expansión que provoca un sector o industria en todo el sistema y la "sensibilidad de dispersión", entendida como la medida de la afectación de un sector cuando aumenta la demanda final de todas las ramas en una unidad (Banguero et al., 2006; Pino \& Illanes, 2002).

\section{Herramientas informáticas de simulación}

Si bien existen varios programas que permiten la construcción por medios de estimación indirectos de una matriz insumo-producto, por ejemplo, Python Module for Input-Output Analysis del REAL (The University of Illinois at UrbanaChampaign, 2017), así como el Simulador de impactos insumo producto 2008 - 2012 del Instituto Nacional de Estadística y Geografía de México - INEGI- (2018); en el análisis de los impactos económicos en la demanda final, la producción y el empleo, inducidos por cambios en los componentes de la demanda final de los sectores económicos priorizados en la distintas alternativas estudiadas en la presente investigación se utilizan herramientas informáticas adaptadas a la especificidad territorial ecuatoriana; particularmente, el simulador desarrollado por el Banco Central del Ecuador (Banco Central del Ecuador, 2017b)

\section{METODOLOGÍA}

La investigación se respalda en la metodología Sistema Europeo de Cuentas (SEC), la cual aborda el análisis de los sistemas económicos desde dos perspectivas, (Carrasco Canals, 1999).

- Análisis Funcional: estudio de la actividad de producción y del equilibrio entre oferta (recursos) y demanda (empleos) de los productos.

- Análisis Estructural: estudio de los modelos económicos que favorezcan la implementación de alternativas óptimas de matriz productiva.

En la fundamentación teórica para el cálculo de la matriz óptima y las proyecciones de los impactos económicos y sociales, consignada en el apartado 
del Marco teórico, se aplican técnicas de búsqueda y gestión de la información científica sustentada en bases de datos y recursos bibliográficos en formatos impresos y digitales.

Para el cálculo de los efectos en la demanda final, la producción y el empleo ocasionados por variaciones en los componentes de la demanda final, utilizando el método insumo-producto, se consideran para la simulación tres priorizaciones de productos estratégicos para el cambio de la matriz productiva del Ecuador. La primera es la que fomenta el Gobierno Nacional 2013 - 2017 con 14 sectores y 5 industrias estratégicos, que corresponde a 30 productos contemplados en la nomenclatura internacional $\mathrm{CPCN}$; la segunda, del Banco Central del Ecuador, considera la priorización en la publicación de la Matriz Insumo producto 2013, asimismo, de 30 productos identificados como clave, motores e isla; y la tercera es la identificada en los resultados de la encuesta de percepción aplicada a la ciudadanía y sectores productivos del Ecuador por los autores de la presente investigación en el año 2016, se priorizan también 30 productos $^{8}$ con el fin de homogeneizar la línea de base para las tres alternativas. (Ver Anexo 1). La herramienta de simulación empleada es la del Banco Central del Ecuador (Banco Central del Ecuador, 2017b).

Se realiza una primera simulación en la que se incrementa la demanda final

\footnotetext{
8 La encuesta aplicada a la Ciudadanía a nivel nacional contempla una muestra de 384 personas de una población de 16'528.730 habitantes (proyectada al 2016) (INEC, 2017); para el cálculo del tamaño de la muestra se considera el $5 \%$ de error, Nivel de Confianza 95\%, Varianza 0.25, Valor tipificado "z" de 1,96 a doble cola. La encuesta al sector empresarial a nivel nacional se aplica a una muestra de 149 empresas que cumplimentaron la misma, de un total de 843.644 (INEC, 2017)
}

actual en un $10 \%$ en cada uno de los 30 productos correspondientes a cada una de las tres priorizaciones antes mencionadas, particularmente en los componentes de exportaciones y consumo interno; en una segunda se reduce la demanda final en $10 \%$, asimismo en cada uno de los 30 productos correspondientes a cada una de las tres priorizaciones; particularmente en los componentes de exportaciones y consumo interno. En el primer caso se establece el supuesto que el país podría obtener recursos adicionales para inversión hasta en un 10\% por ejemplo gracias al incremento de los precios del petróleo, inversión extranjera directa o inversión de ahorro privado; y en el segundo, se considera un escenario en el cual la crisis del país se profundiza y la reducción en la demanda final puede alcanzar hasta el $10 \%$.

En las tres alternativas coincide la priorización de 12 de los 30 productos seleccionados en cada caso, estos son: 1) Carne, productos de la carne y subproductos, 2) Camarón elaborado, 3) Cacao elaborado, chocolate y productos de confitería, 4) Productos alimenticios diversos, 5) Hilos, hilados; tejidos y confecciones, 6) Prendas de vestir, 7) Cuero, productos de cuero y calzado, 8) Productos de madera tratada, corcho y otros material, 9) Electricidad, 10) Servicios de alojamiento, 11) Servicios de restaurante, 12) Servicios de telecomunicaciones, transmisión e información. (Ver Anexo 1).

De la información que genera el simulador del Banco Central del Ecuador, para el análisis objeto de este estudio, se toman en cuenta los siguientes resultados: 1) demanda final, 2) valor inicial de la producción, 3) empleo situación inicial, 4) efecto directo total en la demanda final, 5) valor final de la producción, 6) incremento ab- 
Tabla $N^{\circ} 1$ Resultados de la simulación con el $10 \%$ de incremento en la demanda final ${ }^{10}$

\begin{tabular}{|c|c|c|c|c|c|c|c|c|c|}
\hline 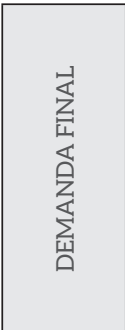 & 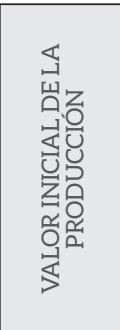 & 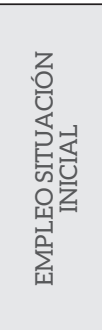 & 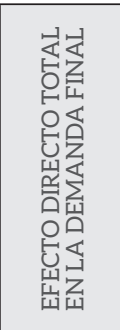 & 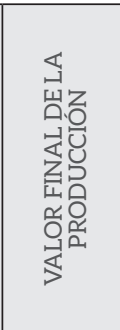 & 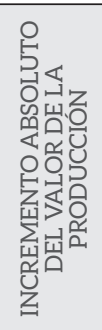 & 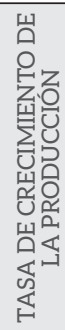 & 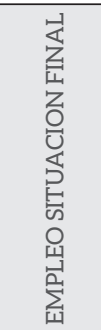 & 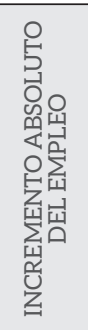 & 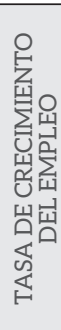 \\
\hline \multicolumn{10}{|c|}{ (Incremento de 10\% en la demanda final) -Priorización del Gobierno Nacional 2013 - 2017.} \\
\hline 106.445 .901 & 161.144 .967 & 7.021 .497 & 112.310 .344 & 170.502 .762 & 9.357.795 & 390 & 7.375 .936 & 354.439 & 390 \\
\hline \multicolumn{10}{|c|}{ (Incremento del 10\% en la demanda final) -Priorización Banco Central del Ecuador (Rasmussen). } \\
\hline 106.445 .901 & 161.144 .967 & 7.021.497 & 108.710 .286 & 165.407 .837 & 4.262 .870 & 319 & 7.231.251 & 209.754 & 319 \\
\hline \multicolumn{10}{|c|}{ (Incremento 10\% en la demanda final) -Priorización Encuesta Nacional de percepción ciudadana y sectores productivos. } \\
\hline 106.445 .901 & 161.144.967 & 7.021.497 & 112.105.760 & 169.858.931 & 8.713.964 & 366 & 7.414.068 & 392.571 & 366 \\
\hline
\end{tabular}

Elaborado por: Los autores.

soluto del valor de la producción, 7) tasa de crecimiento de la producción, 8) empleo situación final, 9) incremento absoluto del empleo, 10) tasa de crecimiento del empleo.

Finalmente, se analizan las implicaciones de las alternativas de priorización de los sectores estratégicos para el cambio de la matriz productiva del Ecuador; esto es, la priorización del Gobierno Nacional (20122017), del Banco Central del Ecuador MIP 2013, y de los resultados de la Encuesta de percepción nacional realizada como parte de este estudio. Este análisis permite probar si la priorización de los sectores económicos que realiza el Gobierno Nacional esóptima ${ }^{\circ}$ o sepuedenidentificar otras propuestas de priorización con resultados mucho mejores, como por ejemplo la del Banco Central del Ecuador identificada en la publicación de la MIP 2013, o la resultante de la aplicación de la Encuesta nacional a la ciudadanía y sectores productivos del Ecuador

9 De acuerdo a Hillier y Lieberman .(Hillier \& Lieberman, 2002) una característica de la investigación de operaciones es que intenta encontrar una mejor solución -llamada solución óptima-Se dice una mejor solución y no la mejor solución porque es posible que existan muchas soluciones que puedan considerarse como las mejores.

\section{RESULTADOS Y DISCUSIÓN}

Los resultados de la simulación en la demanda final, la producción y en el empleo si se incrementa en un 10\% la demanda final, se visualizan en la tabla 1:

Se observa que el mayor impacto positivo del incremento del $10 \%$ en la demanda final y en el valor final de la producción corresponde a la simulación de la priorización del Gobierno Nacional (5.51\% y 5.81\% respectivamente); seguido por la priorización resultante de la Encuesta nacional de percepción ciudadana y de los sectores productivos (incremento en la demanda final de $5.32 \%$ y de $5.41 \%$ en el valor final de la producción); mientras que la priorización resultante de la Encuesta nacional de percepción genera un mayor impacto en el empleo (incremento del 5.59\%) con respecto a la del Gobierno Nacional (5.05\% de incremento). En tercer lugar se ubica el impacto de la priorización del Banco Central del Ecuador, (incremento de $2.13 \%$ en la demanda final, $2.65 \%$ en el valor final de la producción y $2.99 \%$ en el empleo).

10 En miles de dólares americanos para los valores correspondientes a la demanda final y a la producción. 
Gráfico 1: Resultados de la simulación con el 10\% de incremento en la demanda final.

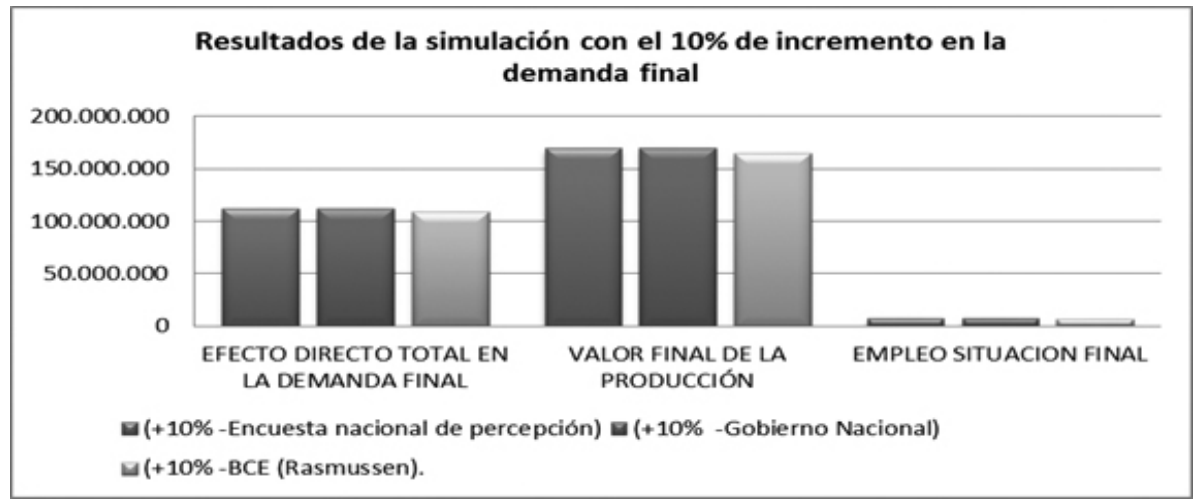

Elaborado por: Los autores.

Evidentemente, los resultados de la priorización del Gobierno Nacional con la de la Encuesta de percepción ciudadana y de los sectores productivos no tienen diferencias porcentuales significativas; con respecto a la priorización del Banco Central del Ecuador se nota cierta diferencia porcentual, que en términos absolutos tampoco es muy amplia.

Los resultados de la simulación en la demanda final, la producción y en el empleo si se reduce en un $10 \%$ la demanda final, se visualizan en la tabla 2.

Tabla $\mathbf{N}^{\circ} 2$ Resultados de la simulación con el 10\% de reducción en la demanda final ${ }^{11}$

\begin{tabular}{|c|c|c|c|c|c|c|c|c|c|}
\hline $\begin{array}{l}\text { 峞 } \\
\text { 岕 } \\
\text { 岕 } \\
\text { 岕 } \\
\text { 岕 }\end{array}$ & 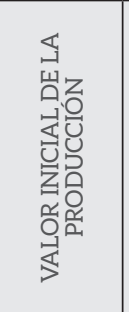 & 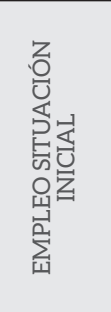 & 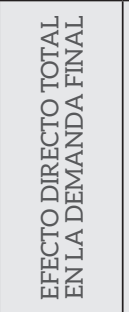 & 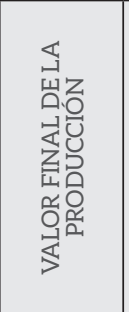 & 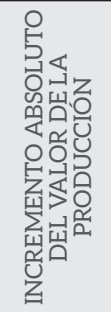 & 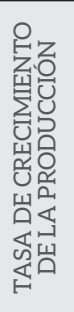 & 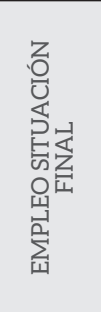 & 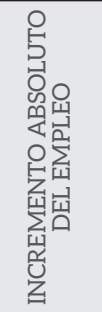 & 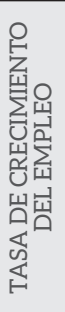 \\
\hline \multicolumn{10}{|c|}{ (Incremento de 10\% en la demanda final) -Priorización del Gobierno Nacional 2013 - 2017.} \\
\hline 106.445 .901 & 161.144 .967 & 7.021 .497 & 100.505 .203 & 151.662 .840 & -9.482 .127 & -402 & 6.653 .255 & -368.242 & -402 \\
\hline \multicolumn{10}{|c|}{ (Reducción -10\% en la demanda fina) -Priorización Banco Central del Ecuador (Rasmussen). } \\
\hline 106.445 .901 & 161.144 .967 & 7.021 .497 & 104.100 .242 & 156.722 .418 & -4.422 .549 & -328 & 6.794 .020 & -227.477 & -328 \\
\hline \multicolumn{10}{|c|}{ (Reducción -10\% en la demanda final) -Priorización Encuesta Nacional de percepción ciudadana y sectores productivos. } \\
\hline 106.445.901 & 161.144 .967 & 7.021 .497 & 100.417 .586 & 151.887 .015 & -9.257 .952 & -380 & 6.617.878 & -403.619 & -380 \\
\hline
\end{tabular}

Elaborado por: Los autores.

En la tabla anterior se tiene que la mayor reducción en la demanda final disminuyendo en el $10 \%$ con respecto a los valores iniciales de la demanda final alcanza la priorización de la Encuesta

11 En miles de dólares americanos para los valores correspondientes a la demanda final y a la producción. nacional (-5.66\%), seguida de la del Gobierno Nacional (-5.58\%) y luego la priorización del Banco Central del Ecuador (-2.20\%). En cuanto al efecto negativo en el valor final de la producción el mayor nivel de disminución ocurre con la priorización del Gobierno Nacional (5.88\%), luego la de 
la Encuesta nacional (-5.75\%) y en tercer lugar la priorización del Banco Central del Ecuador (-2.74\%). En lo relacionado con el impacto negativo en el empleo, la mayor disminución se alcanza con la priorización de la Encuesta nacional (-5.75\%), luego con la del Gobierno Nacional (-5.24\%) y finalmente con el menor impacto negativo la priorización del Banco Central del Ecuador (-3.24\%).

Gráfico 2: Resultados de la simulación con el 10\% de reducción en la demanda final.

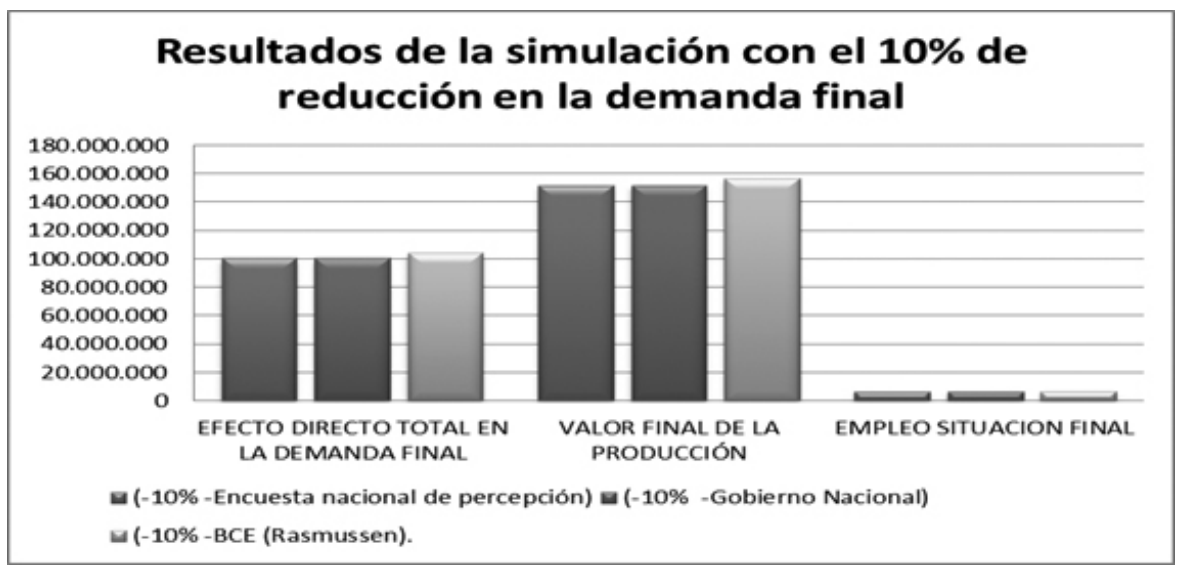

Elaborado por: Los autores.

\section{CONCLUSIONES Y TRABAJO A FUTURO}

- El análisis de los impactos económicos inducidos por cambios en la demanda final de los sectores estratégicos para el cambio de la matriz productiva del Ecuador permite concluir que si bien la alternativa de priorización de los sectores económicos que realiza el Gobierno Nacional es la óptima en cuanto al efecto directo total en la demanda final y en el valor final de la producción, no lo es en cuanto al incremento en el nivel de empleo; siendo la priorización óptima, para este caso, la resultante de la Encuesta nacional aplicada a la ciudadanía y sectores productivos del país. Se determina que el menor impacto en los indicadores antes indicados resulta de la priorización realizada en la publicación de la Matriz Insumo Producto 2013 del Banco Central del Ecuador.
- Evidentemente la diferencia porcentual entre las dos primeras priorizaciones, incluso con la tercera señalada, no es significativa; consecuentemente las tres metodologías empleadas en las alternativas de priorización pueden ser válidas para priorizar sectores clave para el cambio de la matriz productiva del Ecuador; esto es: 1) la metodología participativa contemplada en la Ley Orgánica de Participación Ciudadana y en criterios relacionados con la potencialidad de los sectores priorizados para aprovechar la política de sustitución de importaciones y del comercio exterior; 2) la identificación de sectores clave, motor y base utilizando el método de los coeficientes de Rasmussen; y, 3) la aplicación del muestreo estadístico sustentado en técnicas e instrumentos de investigación científica.

- Se cuenta con una herramienta de simulación informatizada validada que utiliza las tablas Insumo - Producto 
adaptadas a la especificidad ecuatoriana y que contribuye a tomar mejores decisiones en cuanto a la inversión pública y privada en función de los sectores productivos con mayor aporte al incremento de los indicadores tales como la demanda final, la producción y el empleo; consecuentemente se tiene la posibilidad de generar políticas públicas que fortalezcan y estimulen la inversión nacional y extranjera a favor de la productividad y competitividad e la economía ecuatoriana.
- El estudio abre las puertas para que en futuras investigaciones se contrasten los resultados obtenidos con la aplicación de modelos multisectoriales y simuladores basados en la Matriz de Contabilidad Social y en modelizaciones del Modelo de Equilibrio General Aplicado, y así contar con más elementos de juicio para la toma de decisiones de inversión y de políticas públicas vinculadas con el desarrollo sostenible del país.

\section{REFERENCIAS BIBLIOGRÁFICAS}

Aroche, F., Frías, S., \& Torres, L. (2012, April). Matriz de insumo producto para América del Norte. Revista Realidad, Datos Y Espacio. Revista Internacional de Estadística Y Geografía, 70-89. Retrieved from http://www.inegi.org.mx/prod_serv/contenidos/espanol/bvinegi/productos/ integracion/especiales/revist-inter/revista_5/RDE_05_4a.html

Asamblea Nacional del Ecuador. (2011). Ley Orgánica de Participacion Ciudadana. Quito: Lexis. Retrieved from http://www.oas.org/juridico/pdfs/mesicic4_ecu_org6.pdf

Banco Central de Chile. (2001). Matriz de Insumo Producto de la Economía Chilena 1996. Retrieved from http://si3.bcentral.cl/estadisticas/Principal1/Informes/CCNN/cdr/matriz1996.pdf

Banco Central de Venezuela. (2012). Matriz Insumo Producto. Retrieved February 17, 2016, from http://www.bcv.org.ve/cuadros/series/mip97/mip97.asp?id=425

Banco Central del Ecuador. (2011). Cuentas Nacionales. Cuadernos de Trabajo. Quito: Banco Central del Ecuador. http://doi.org/10.1016/S0210-0266(11)70011-5

Banco Central del Ecuador. (2014). Matriz Insumo Producto: Simétrica e Inversa. Retrieved from http:// contenido.bce.fin.ec/documentos/PublicacionesNotas/Catalogo/CuentasNacionales/Anuales/ Dolares/PR_MatrizInsumoProducto.pdf

Banco Central del Ecuador. (2017a). Base de datos del Banco Central del Ecuador. Retrieved from https://www.bce.fin.ec/

Banco Central del Ecuador. (2017b). bce.fin.ec. Retrieved September 13, 2017, from https://www.bce. fin.ec/

Banderas, V., \& Hidalgo, A. (2013). Análisis de impacto en la economía ecuatoriana por shocks exógenos en el sector agrícola (mediante el método input - output para el año 2007. Escuela Politécnica Nacional. Retrieved from http://bibdigital.epn.edu.ec/handle/15000/6014

Banguero, H., Duque, H., Garizado, P., \& Parra, D. (2006). Estimación de la matriz insumo producto simétrica para el Valle del Cauca - año 1994. Calí. Retrieved from http://www.libreriadelau. com/estimacion-de-la-matriz-insumo-producto-simetrica-para-el-valle-del-cauca-ano-1994sociologia-sociedad-y-cultura.html 
Campoverde, M., \& López, C. (2014). Elaboración de la matriz insumo producto y cálculo de los coeficientes de Rasmussen para la provincial del Cañar para el año 2007. Universidad de Cuenca. Retrieved from http://dspace.ucuenca.edu.ec/handle/123456789/5565

Carrasco Canals, F. (1999). Fundamentos del sistema europeo de cuentas nacionales y regionales (SEC 1995). Madrid U6.

Departamento Nacional de Estadística de Colombia. (2014). Ficha Metodológica Cuentas Anuales de Bienes y Servicios - CABYS--. Retrieved from http://www.dane.gov.co/files/investigaciones/ fichas/pib/Ficha_met_ctas_anua_bie_ser_03_14.pdf

Fernández, N. (2007). Análisis input-output: identificación de los encadenamientos productivos y los sectores clave de la economía ecuatoriana para el año 2007. Facultad Latinoamericana de Ciencias Sociales - Sede Ecuador. Retrieved from http://repositorio.flacsoandes.edu.ec/ handle/10469/1980\#.WFMQvdJ97IU

Flores-Tapia, C., \& Flores-Cevallos, L. (2016). Herramientas para simulación de matrices productivas óptimas del Ecuador. Economía \& Administración, 7(2), 101-112. Retrieved from http://www.iiesunah.org/Revista/index.php/EyA/article/view/405/pdf

Hernández Sampieri, R., Fernández Collado, C., \& Baptista Lucio, P. (2007). Fundamentos de metodología de la investigación. Madrid: McGraw-Hill.

Hillier, F., \& Lieberman, G. (2002). Investigación de operaciones. México [etc.]: McGraw-Hill/ Interamericana.

INEC. (2017). Ecuadorencifras. Retrieved from http://www.ecuadorencifras.gob.ec/indice-verdeurbano/

Instituto Nacional de Estadística y Censos Argentina. Ministerio de Economía. Secretaría de Política Económica. (2001). Matriz insumo producto Argentina 1997.

Instituto Nacional de Estadística y Geografía de México. (2018). Simulador de Impactos de Insumo Producto. Retrieved from http://www.inegi.org.mx/est/contenidos/proyectos/cn/mip12/ simulador.aspx

Lind, D. (2012). Estadistica aplicada a los negocios y la economía. Estadistica Aplicada a Los Negocios Y La Economía, 15(2), 81-87. http://doi.org/10.1007/s13398-014-0173-7.2

Ministerio Coordinador de la Producción, E. y C. (2010). Agenda para la transformación productiva. Quito: Ministerio de Coordinación de la Producción, Empleo y Competitividad.

Nobel Prize. (2016). Wassily Leontief - Biographical. Retrieved February 18, 2016, from http://www. nobelprize.org/nobel_prizes/economic-sciences/laureates/1973/leontief-bio.html

Pino, O., \& Illanes, W. (2002). Análisis exploratorio de los coeficientes de Rasmussen para la economía regional, mediante la utilización de las tablas input - output para la economía chilena, base 1996. Theoria, 11, 69-76. Retrieved from http://docplayer.es/22945562-Theoria-vol-11-69-76-2002issn-0717-196x.html

Sancho, F., \& Cardenete, M. A. (2014a). Instrumentos multisectoriales para la detección de sectores clave en el análisis regional. Revista de Estudios Regionales, (100), 131-146. Retrieved from http:// www.scopus.com/inward/record.url?eid=2-s2.0-84921474905\&partnerID=tZOtx3y1 
Sancho, F., \& Cardenete, M. A. (2014b). Instrumentos multisectoriales para la detección de sectores clave en el análisis regional. Revista de Estudios Regionales, 7585(100), 131-146.

Secretaría Nacional de Planificación y Desarrollo. (2012). Transformación de la Matriz Productiva. SENPLADES. Retrieved from http://www.planificacion.gob.ec/wp-content/uploads/ downloads/2013/01/matriz_productiva_WEBtodo.pdf

Tarancón Morán, M. Á. (2011). Técnicas de análisis económico input-output. ECU.

The University of Illinois at Urbana-Champaign. (2017). Regional Economics Applications Laboratory. Retrieved from http://www.real.illinois.edu/realio/

\section{Para citaciones:}

Flores-Tapia, C. E., \& Flores-Cevallos, K. L. (2017). Impactos económicos inducidos por la demanda final en sectores estratégicos para el cambio de la estructura productiva del Ecuador. Panorama Económico, 25, 3, pp. 443-458.

\section{AUTORES}

Carlos Ernesto Flores-Tapia

Profesor e Investigador de la Pontificia Universidad Católica del Ecuador. Actualmente cursa estudios doctorales en Ciencias Sociales y Jurídicas de la Universidad de Cádiz (España)

Karla Lissette Flores-Cevallos

Investigadora y actualmente directora de proyectos de la Fundación Los Andes en Ecuador. Cuenta con estudios de posgrado en Economía y Desarrollo Territorial de la Universidad de Cadiz (España). 


\section{Anexo 1: Productos seleccionados en cada alternativa de priorización ${ }^{*}$}

\begin{tabular}{|c|c|c|c|c|c|c|}
\hline $\mathrm{N}^{\circ}$ & $\mathrm{CPCN}$ & $\begin{array}{c}\text { Priorización del } \\
\text { Gobierno Nacional } 2013 \\
-2017 .\end{array}$ & $\mathrm{CPCN}$ & $\begin{array}{c}\text { Priorización Banco } \\
\text { Central del Ecuador } \\
\text { (Rasmussen). }\end{array}$ & $\mathrm{CPCN}$ & $\begin{array}{c}\text { Priorización Encuesta } \\
\text { Nacional de percepción } \\
\text { ciudadana y sectores } \\
\text { productivos. }\end{array}$ \\
\hline 1 & 001001 & 1 Banano, café y cacao & 005001 & $\begin{array}{l}7 \text { Animales vivos y } \\
\text { productos animales }\end{array}$ & 1001 & 1 Banano, café y cacao \\
\hline 2 & 002001 & 2 Cereales & 007001 & $\begin{array}{l}\text { 9 Camarón vivo o fresco } \\
\text { y larvas de camarón }\end{array}$ & 003001 & 3 Flores y capullos \\
\hline 3 & 004001 & $\begin{array}{l}4 \text { Tubérculos, Vegetales, } \\
\text { melones y frutas }\end{array}$ & 011001 & $\begin{array}{l}16 \text { Carne, productos de la } \\
\text { carne y subproductos }\end{array}$ & 011001 & $\begin{array}{l}16 \text { Carne, productos de } \\
\text { la carne y subproductos }\end{array}$ \\
\hline 4 & 008001 & $\begin{array}{l}10 \text { Pescado y otros } \\
\text { productos acuáticos } \\
\text { (excepto camarón) }\end{array}$ & 012001 & 17 Camarón elaborado & 012001 & 17 Camarón elaborado \\
\hline 5 & 009001 & $\begin{array}{l}12 \text { Petróleo crudo y gas } \\
\text { natural }\end{array}$ & 013001 & $\begin{array}{l}18 \text { Pescado y otros } \\
\text { productos acuáticos } \\
\text { elaborados }\end{array}$ & 018001 & $\begin{array}{l}26 \text { Cacao elaborado, } \\
\text { chocolate y productos } \\
\text { de confitería }\end{array}$ \\
\hline 6 & 011001 & $\begin{array}{l}16 \text { Carne, productos de la } \\
\text { carne y subproductos }\end{array}$ & 014001 & $\begin{array}{l}20 \text { Aceites crudos y } \\
\text { refinados }\end{array}$ & 19003 & $\begin{array}{l}29 \text { Productos } \\
\text { alimenticios diversos }\end{array}$ \\
\hline 7 & 012001 & 17 Camarón elaborado & 015001 & $\begin{array}{l}21 \text { Productos lácteos } \\
\text { elaborados }\end{array}$ & 021001 & $\begin{array}{l}33 \text { Hilos, hilados; tejidos } \\
\text { y confecciones }\end{array}$ \\
\hline 8 & 013001 & $\begin{array}{l}18 \text { Pescado y otros } \\
\text { productos acuáticos } \\
\text { elaborados }\end{array}$ & 016001 & $\begin{array}{l}22 \text { Productos de } \\
\text { molinería }\end{array}$ & 021002 & 34 Prendas de vestir \\
\hline 9 & 013002 & $\begin{array}{l}19 \text { Preparados y } \\
\text { conservas de pescado } \\
\text { y de otras especies } \\
\text { acuáticas }\end{array}$ & 016002 & $\begin{array}{l}23 \text { Productos de la } \\
\text { panadería }\end{array}$ & 021003 & $\begin{array}{l}35 \text { Cuero, productos de } \\
\text { cuero y calzado }\end{array}$ \\
\hline 10 & 015001 & $\begin{array}{l}21 \text { Productos lácteos } \\
\text { elaborados }\end{array}$ & 016003 & $\begin{array}{l}24 \text { Fideos, macarrones } \\
\text { y otros productos } \\
\text { farináceos similares }\end{array}$ & 022001 & $\begin{array}{l}36 \text { Productos de madera } \\
\text { tratada, corcho y otros } \\
\text { material }\end{array}$ \\
\hline 11 & 016002 & $\begin{array}{l}23 \text { Productos de la } \\
\text { panadería }\end{array}$ & 017001 & $\begin{array}{l}25 \text { Azúcar, panela y } \\
\text { melaza }\end{array}$ & 025001 & $\begin{array}{l}39 \text { Productos químicos } \\
\text { básicos, abonos y } \\
\text { plásticos primarios }\end{array}$ \\
\hline 12 & 016003 & $\begin{array}{l}24 \text { Fideos, macarrones } \\
\text { y otros productos } \\
\text { farináceos similares }\end{array}$ & 018001 & $\begin{array}{l}26 \text { Cacao elaborado, } \\
\text { chocolate y productos de } \\
\text { confitería }\end{array}$ & 025002 & $\begin{array}{l}40 \text { Otros productos } \\
\text { químicos }\end{array}$ \\
\hline 13 & 018001 & $\begin{array}{l}26 \text { Cacao elaborado, } \\
\text { chocolate y productos de } \\
\text { confitería }\end{array}$ & 019002 & $\begin{array}{l}28 \text { Productos de café } \\
\text { elaborado }\end{array}$ & 026001 & 41 Productos de caucho \\
\hline 14 & 019002 & $\begin{array}{l}28 \text { Productos de café } \\
\text { elaborado }\end{array}$ & 019003 & $\begin{array}{l}29 \text { Productos } \\
\text { alimenticios diversos }\end{array}$ & 026002 & $\begin{array}{l}42 \text { Productos de } \\
\text { plástico }\end{array}$ \\
\hline 15 & 019003 & $\begin{array}{l}29 \text { Productos } \\
\text { alimenticios diversos }\end{array}$ & 020001 & 30 Bebidas alcohólicas & 030001 & $\begin{array}{l}48 \text { Equipo de } \\
\text { transporte }\end{array}$ \\
\hline 16 & 021001 & $\begin{array}{l}33 \text { Hilos, hilados; tejidos } \\
\text { y confecciones }\end{array}$ & 020002 & 31 Bebidas no alcohólicas & 032001 & $\begin{array}{l}50 \text { Otros productos } \\
\text { manufacturados }\end{array}$ \\
\hline 17 & 021002 & 34 Prendas de vestir & 020003 & 32 Tabaco elaborado & 033001 & 51 Electricidad \\
\hline
\end{tabular}

\footnotetext{
* La nomenclatura de los 30 productos seleccionados en cada una de las alternativas de priorización se toman de la clasificación del Código Central de Productos Nacional (CPC19N) de la Matriz Insumo Producto (Resultados del año 2013) publicada por el Banco Central del Ecuador. Matriz de 71 x 71 productos (Banco Central del Ecuador, 2017b). CPCN (Banco Central del Ecuador, 2011)
} 


\begin{tabular}{|c|c|c|c|c|c|c|}
\hline 18 & 021003 & $\begin{array}{l}35 \text { Cuero, productos de } \\
\text { cuero y calzado }\end{array}$ & 021001 & $\begin{array}{l}33 \text { Hilos, hilados; tejidos } \\
\text { y confecciones }\end{array}$ & 033002 & $\begin{array}{l}52 \text { Agua, servicios de } \\
\text { saneamiento y gas } \\
\text { (excepto de petróleo) }\end{array}$ \\
\hline 19 & 022001 & $\begin{array}{l}36 \text { Productos de madera } \\
\text { tratada, corcho y otros } \\
\text { material }\end{array}$ & 021002 & 34 Prendas de vestir & 034001 & $\begin{array}{l}53 \text { Trabajos de } \\
\text { construcción y } \\
\text { construcción }\end{array}$ \\
\hline 20 & 024001 & $\begin{array}{l}38 \text { Aceites refinados } \\
\text { de petróleo y de otros } \\
\text { productos }\end{array}$ & 021003 & $\begin{array}{l}35 \text { Cuero, productos de } \\
\text { cuero y calzado }\end{array}$ & 035002 & $\begin{array}{l}55 \text { Servicios de } \\
\text { reparación y } \\
\text { mantenimiento de } \\
\text { vehículos de motor y } \\
\text { motocicletas }\end{array}$ \\
\hline 21 & 025001 & $\begin{array}{l}39 \text { Productos químicos } \\
\text { básicos, abonos y } \\
\text { plásticos primarios }\end{array}$ & 022001 & $\begin{array}{l}36 \text { Productos de madera } \\
\text { tratada, corcho y otros } \\
\text { material }\end{array}$ & 036001 & $\begin{array}{l}56 \text { Servicios de } \\
\text { alojamiento }\end{array}$ \\
\hline 22 & 025002 & $\begin{array}{l}40 \text { Otros productos } \\
\text { químicos }\end{array}$ & 027002 & $\begin{array}{l}44 \text { Cemento, artículos de } \\
\text { hormigón y piedra }\end{array}$ & 036002 & $\begin{array}{l}57 \text { Servicios de } \\
\text { restaurante }\end{array}$ \\
\hline 23 & 030001 & 48 Equipo de transporte & 028001 & 45 Metales comunes & 037001 & $\begin{array}{l}58 \text { Servicios de } \\
\text { transporte y } \\
\text { almacenamiento }\end{array}$ \\
\hline 24 & 031001 & 49 Muebles & 029001 & $\begin{array}{l}47 \text { Maquinaria, equipo y } \\
\text { aparatos eléctricos }\end{array}$ & 038002 & $\begin{array}{l}60 \text { Servicios de } \\
\text { telecomunicaciones, } \\
\text { transmisión e } \\
\text { información }\end{array}$ \\
\hline 25 & 033001 & 51 Electricidad & 031001 & 49 Muebles & 040001 & $\begin{array}{l}62 \text { Servicios de seguros } \\
\text { y fondos de pensiones }\end{array}$ \\
\hline 26 & 034001 & $\begin{array}{l}53 \text { Trabajos de } \\
\text { construcción y } \\
\text { construcción }\end{array}$ & 033001 & 51 Electricidad & 041001 & $\begin{array}{l}63 \text { Servicios } \\
\text { inmobiliarios }\end{array}$ \\
\hline 27 & 036001 & $\begin{array}{l}56 \text { Servicios de } \\
\text { alojamiento }\end{array}$ & 036001 & $\begin{array}{l}56 \text { Servicios de } \\
\text { alojamiento }\end{array}$ & 044001 & $\begin{array}{l}66 \text { Servicios de } \\
\text { enseñanza privado }\end{array}$ \\
\hline 28 & 036002 & $\begin{array}{l}57 \text { Servicios de } \\
\text { restaurante }\end{array}$ & 036002 & $\begin{array}{l}57 \text { Servicios de } \\
\text { restaurante }\end{array}$ & 044002 & $\begin{array}{l}67 \text { Servicios de } \\
\text { enseñanza público (no } \\
\text { de mercado) }\end{array}$ \\
\hline 29 & 037001 & $\begin{array}{l}58 \text { Servicios de } \\
\text { transporte y } \\
\text { almacenamiento }\end{array}$ & 038001 & $\begin{array}{l}59 \text { Servicios postales y de } \\
\text { mensajería }\end{array}$ & 045001 & $\begin{array}{l}68 \text { Servicios sociales y } \\
\text { de salud privado }\end{array}$ \\
\hline 30 & 038002 & $\begin{array}{l}60 \text { Servicios de } \\
\text { telecomunicaciones, } \\
\text { transmisión e } \\
\text { información }\end{array}$ & 038002 & $\begin{array}{l}60 \text { Servicios de } \\
\text { telecomunicaciones, } \\
\text { transmisión e } \\
\text { información }\end{array}$ & 045002 & $\begin{array}{l}69 \text { Servicios sociales y } \\
\text { de salud no de mercado }\end{array}$ \\
\hline
\end{tabular}

Elaborado por: Los autores. 